\title{
Corela
}

Cognition, représentation, langage

HS-8 | 2010

L'interpellation

\section{Interpellation et enjeux de pouvoir dans les comédies et tragédies latines}

Sophie Roesch

\section{OpenEdition}

Journals

Édition électronique

URL : http://journals.openedition.org/corela/1632

DOI : $10.4000 /$ corela.1632

ISSN : $1638-573 \mathrm{X}$

Éditeur

Cercle linguistique du Centre et de l'Ouest - CerLICO

Référence électronique

Sophie Roesch, «Interpellation et enjeux de pouvoir dans les comédies et tragédies latines », Corela [En ligne], HS-8 | 2010, mis en ligne le 02 novembre 2010, consulté le 30 avril 2019. URL : http:// journals.openedition.org/corela/1632; DOI : 10.4000/corela.1632

Ce document a été généré automatiquement le 30 avril 2019.

\section{(c) (i) (2)(2)}

Corela - cognition, représentation, langage est mis à disposition selon les termes de la licence Creative Commons Attribution - Pas d'Utilisation Commerciale - Partage dans les Mêmes Conditions 4.0 International. 


\title{
Interpellation et enjeux de pouvoir dans les comédies et tragédies latines
}

\author{
Sophie Roesch
}

\section{0 . Introduction}

1 Nombreuses sont les interpellations entre personnages dans les comédies et tragédies latines $^{1}$ : interpellations «réelles » en direction d'un autre personnage présent, qui postulent une réponse, interpellations-invocations aux dieux (qui sont convoqués par le locuteur, soit qu'il les prenne à témoin de ce qui lui arrive, soit qu'il sollicite d'eux une faveur, par exemple Amphitryon 923 : summe Iupiter, «très haut Jupiter»), interpellations auto-adressées (ainsi, chez Sénèque, en Médée 937, Médée interpelle son âme : anime ${ }^{2}$; ou chez Plaute, en Asinaria 249, Liban se parle à lui-même: Libane), interpellations rhétoriques adressées à un absent (ainsi en Phèdre $696^{3}$, Hippolyte interpelle son père absent: genitor), ou servant simplement à souligner les différentes directions dans lesquelles est attiré l'esprit du héros qui monologue (cf. chez Sénèque, Hercule Furieux, où Hercule, dans son discours douloureux, convoque successivement son père, la Terre, ses enfants, et enfin ses propres mains meurtrières v.1202-1236).

2 Notre communication sera axée sur le premier type d'interpellations : celui qui a lieu entre personnages. Dans un corpus théâtral, il ne faut pas oublier que le choix d'un appellatif n'est jamais neutre, car il permet d'informer efficacement et économiquement le public des relations de " place $»^{4}$ entre les personnages.

3 Au-delà de cette fonction informative vis-à-vis du public, mais aussi vis-à-vis de l'interlocuteur à qui il est bon parfois de rappeler sa "place» (surtout quand c'est un supérieur qui s'adresse à un inférieur), les termes d'adresse jouent un rôle essentiel au sein de l'échange pour entretenir le lien conversationnel. Comme le souligne Détrie (2006, 92), «l'apostrophe, en tant qu'unité conversationnelle produite par le locuteur a une double fonction: au-delà de sa fonction première de ratification de l'allocutaire, elle révèle surtout une autre visée, qui est d'accentuer le lien interlocutif. Ce marquage 
interpersonnel à visée phatique est à envisager comme la manifestation langagière d'une volonté de maintien du lien conversationnel, et l'apostrophe est alors une expression de la pulsion communicative, servant de renforçateur de la relation interpersonnelle. "

4 En latin, les interpellations se caractérisent en général par l'emploi d'un cas précis : le vocatif. Sur ces emplois, Serbat ${ }^{5}(1987,9)$ précise bien que la valeur du vocatif est «d'ordre uniquement énonciatif: le vocatif pose expressivement dans le discours le destinataire sélectionné par l'énonceur ». Le nominatif peut aussi, de manière moins fréquente, servir à l'interpellation ${ }^{6}$. Dans l'approche qui est la nôtre aujourd'hui, nous ne ferons pas de différence entre interpellation au vocatif ou au nominatif puisqu'elles ne fonctionnent pas de manière pragmatiquement différente.

$5 \quad$ Nous avons choisi de mettre en perspective les emplois des appellatifs dans la comédie et la tragédie antiques car il s'agit de genres littéraires caractérisés chacun par des codes bien distincts, ce qui va influencer les modalités de l'interpellation.

6 Nous commencerons par présenter les différents types d'apostrophe dans les comédies de Plaute, ainsi que la façon dont ils manifestent les rapports de "place» entre les personnages, avant de présenter de manière contrastive leur fonctionnement dans les tragédies de Sénèque.

\section{Les interpellations dans la comédie latine}

7 Rappelons que la comédie, à l'imitation de ses modèles grecs, met en scène des personnages types (l'esclave, le jeune amoureux, l'épouse acariâtre...) ${ }^{7}$ dont les relations dépendent à la fois de leur position sociale, mais aussi de l'intrigue. Les appellatifs jouent un rôle clef quand il s'agit de définir les liens qui unissent ces différents protagonistes : ils servent de taxèmes de position haute ou basse, pour reprendre les termes de $\mathrm{C}$. KerbratOrecchioni. Comme l'écrit C. Kerbrat-Orecchioni $(1988,193)$, «les pronoms d'allocution (...), les titres, les termes d'adresse et autres expressions appellatives... dans certains types de corpus (ainsi dans les dialogues entre maîtres et valets de la comédie classique) » apparaissent comme « les indices les plus éloquents sans doute du système des places. ${ }^{8}$

8 Au-delà de ces emplois, l'usage du nom propre est primordial dans une perspective dramaturgique: il permet plus aisément au spectateur d'identifier, dès la scène d'exposition, qui sont les personnages en scène et, dans le cas d'une conversation entre plusieurs personnages, qui est visé par telle ou telle réplique. Par exemple, en Capt 138-139, Hégion rencontre son parasite ; ils se saluent ce qui permet d'indiquer à peu de frais leur identité :

Heg : Ergasile, salue.

Erg : Di te bene ament, Hegio.

«- Ergasile, salut.

- Que les dieux te bénissent, Hégion. »

9 Nous allons faire l'inventaire des différentes catégories d'appellatifs en nous demandant en quoi ils répondent à la problématique des rapports de "place». Notre analyse s'appuiera aussi sur les notions de «Face Threatening Act » (FTA) (Brown et Levinson, 1987, 65) et de «Face Flattering Act » (FFA) (Kerbrat Orecchioni, 2000, 24) qui permettent de caractériser finement les rapports de pouvoir entre les interlocuteurs. 


\subsection{L'interpellation par un nom propre : essentiellement dans les séquences d'ouverture}

10 Ce sont les noms propres qui sont le plus fréquemment utilisés dans la comédie pour interpeller l'interlocuteur. Cela n'a rien de surprenant puisque, s'il faut en croire Dickey (2002, 43), le nom propre est, en latin, l'appellatif le plus neutre quand il s'agit d'interpeller quelqu'un, - à condition bien sûr que l'on connaisse son identité.

\subsubsection{Avec un rôle de « summons"}

11 Le nom propre joue tout d'abord un rôle de « summons $»^{9}:$ il permet d'interpeller l'autre pour l'intégrer dans la conversation. En Au 199-200, Mégadore essaie d'attirer l'attention d'Euclion qui ne l'écoute guère :

Meg : Da mihi operam parumper; paucis, Euclio, est quod te uolo

De communi re appellare mea et tua.

«Prête moi un tout petit peu attention; Euclion, c'est rapidement que je veux te parler d'une affaire commune à toi et moi.»

\subsubsection{Dans les salutations}

12 Le nom propre est aussi très fréquent dans les séquences d'ouverture du dialogue, au moment des salutations. Par exemple, en Au 182-183 ${ }^{10}$, Euclion et Mégadore se saluent :

Meg : Saluus atque fortunatus, Euclio, semper sies !

Euc : Di te ament, Megadore. (...)

«- Puisses-tu toujours être en bonne santé et fortuné, Euclion!

- Que les dieux t'aiment, Mégadore!»

13 L'énoncé du nom de l'interlocuteur fait partie d'un rituel de prise de contact, et d'ailleurs, il fonctionne souvent sur le modèle des " adjacency pairs », dégagé par Shegloff et Sacks $(1973,295)$. Il s'agit d'un rituel confirmatif qui permet de faciliter la prise de contact entre les interlocuteurs, en leur donnant l'occasion de manifester leur accord quant au début de la discussion.

14 Le nom propre remplit dans ces différentes occurrences une double fonction: appeler l'autre s'il n'a pas vu que le locuteur voulait initier un dialogue, mais aussi l'accueillir dans le dialogue en lui reconnaissant son identité1 ${ }^{11}$. On retrouve là la double fonction que reconnaît Perret (1968) au vocatif ${ }^{12}$ : d'une part appellative, de l'autre phatique. En accueillant autrui par son nom, le locuteur ratifie en quelque sorte son droit à participer à l'échange. Il s'agit d'adoucir le FTA potentiel que constitue la prise de contact

\subsubsection{Pour s'attirer la bienveillance de l'autre}

15 Les noms propres sont par ailleurs fréquemment associés à des actes directifs ou à des questions ${ }^{13}$. Ces modes d'adresse visent à inciter l'interlocuteur à agir ou à parler. Pour augmenter ses chances d'obtenir ce qu'il demande, le locuteur tente de créer une plus grande proximité en recourant au nom propre. Au-delà de ce rôle d'amadouage, le vocatif s'explique aussi parce qu'il vient appuyer et renforcer un acte de langage qui se veut tout particulièrement orienté vers l'interlocuteur.

Par exemple, en Ba 497 à 499, Philoxène donne des ordres aux personnages présents ${ }^{14}$ : 
$\mathrm{Ph}:$ (...) Mnesiloche, cura et concastiga hominem probe

Qui dedecorat te, me, amicos atque alios flagitiis suis.

In te ego hoc onus omne impono. Lyde, sequere hac me.

« (...) Mnésiloque, occupe-t-en et réprimande au mieux celui qui déshonore à la fois

toi, moi, ses amis et d'autres encore, par sa débauche. Je me repose sur toi de cette

charge. Lydus, suis-moi par là. »

On rencontre encore des noms propres dans les contextes où le locuteur cherche à convaincre son interlocuteur, et est donc dans une logique de captatio beneuolentiae. Ainsi, en Capt 547-623, Tyndare tente de persuader Hégion qu'il est un homme libre, du nom de Philocrate, et non un esclave. La situation est tendue car Hégion se heurte à un contradicteur en la personne du jeune Aristophonte. Tyndare va employer cinq fois le nom d'Hégion pour appuyer ses dires. De son côté, Aristophonte, qui veut lui aussi obtenir gain de cause, va recourir quatre fois au nom de ce dernier.

Le nom propre peut, de manière un peu similaire, s'employer de manière phatique à l'appui d'un aveu ou d'une confidence ${ }^{15}$, pour souligner le lien qui unit les interlocuteurs et mieux disposer le confident à écouter ce qu'on va lui dire. Ainsi, en As $60^{16}$, le vieux Déménète se plaint de sa femme auprès de l'oreille compatissante de son esclave :

De : Verum meam uxorem, Libane, nescis qualis sit.

"Mais ma femme, Liban, tu ne sais pas comme elle est !"

Le choix du nom propre peut enfin s'expliquer par des raisons stratégiques. C'est le cas en Ba 121, 139, 137 où son usage relève d'une volonté de nier un lien social hiérarchique entre les locuteurs. Appeler l'autre par son titre ou sa fonction constitue une mise à distance plus grande que si l'on utilise son nom. Pistoclère, qui a été pris en flagrant délit de débauche par son pédagogue, son précepteur, Lydus, tente de se justifier auprès de lui. Il l'appelle par son nom, au lieu de se référer à son statut de pédagogue; comme celui-ci le fait bien remarquer au v. 138, c'est une volonté délibérée de nier le respect qui lui est dû :

Pi : (...) Odiosus mihi es.

Tace atque sequere, Lyde, me.

Ly : Illuc sis uide ;

Non pædagogum iam me, sed Lydum uocat! (Ba 136-138)

«- Tu es importun. Tais-toi et suis-moi, Lydus.

- Voyez ce qui se passe là, s'il vous plaît : il ne m'appelle plus «son pédagogue ", mais « Lydus ». »

\subsection{L'interpellation par un pronom personnel de la deuxième personne}

Le pronom de la deuxième personne du singulier, tu, est l'appellatif par excellence. Quand il s'emploie à l'appui d'une injonction, c'est pour souligner le destinataire de l'acte directif ${ }^{17}$. Rappelons qu'en latin, l'emploi du pronom personnel sujet est marqué puisque la désinence verbale suffit à indiquer la référence à la personne. L'expression du pronom sujet est donc généralement emphatique. Dans les occurrences considérées ici, nous relevons deux raisons à son emploi, plutôt qu'à celui du nom propre.

Tout d'abord, quand le locuteur ignore le nom de l'interlocuteur, par exemple pour s'adresser à des esclaves indéterminés dont le nom n'importe pas à l'action. Ainsi, en Truc 788, Calliclès interroge une servante et une coiffeuse qui ont soustrait l'enfant de sa fille. Il emploie $t u$, sans doute avec un mouvement expressif du visage ou de la main, pour 
désigner celle des deux qu'il souhaite interroger. Il parle tout d'abord à la servante: Loquere, tu (« Parle, toi », Truc 788). Puis, il se tourne vers la coiffeuse : Iam tace. Accepistin puerum tu $a b$ hac? («Maintenant, tais-toi. Toi, as-tu reçu l'enfant des mains de cette femme? », Truc 791).

Mais aussi, il arrive simplement que le locuteur ne juge pas nécessaire d'employer le nom de son interlocuteur. Ce déni du nom propre peut relever d'un souci de ne pas reconnaître l'existence de l'autre comme individu, par exemple quand un maitre s'adresse à un esclave pour lui donner un ordre. Dans ce cas, le choix du pronom personnel est un signe de position haute de la part du maître. Ainsi, en Am 552, Amphitryon s'adresse à son esclave Sosie :

Am : Age i tu secundum (« Allons, toi, suis-moi ») ${ }^{18}$.

Il arrive aussi que le pronom soit complété par une relative déterminative ; l'usage de « tu " relève alors de la volonté de définir l'autre par la relative qui s'y rattache. C'est l'ensemble antécédent + relative qui joue le rôle d'un appellatif, comme en Au 268-269 ${ }^{19}$; l'avare Euclion appelle sa servante qu'il soupçonne de l'avoir trahi :

Euc: Vbi tu es, quae deblaterauisti iam uicinis omnibus Meae me filiae daturum dotem ? heus, Staphyla, te uoco.

«Où es-tu, toi qui a dégoisé à tous mes voisins que je donnerais une dot à ma fille?

Hé, Staphyla, je t'appelle. »

Comme Staphyla ne se reconnaît pas dans cette définition et ne répond pas à cette interpellation, Euclion est obligé de s'adresser à elle de nouveau, cette fois avec un nom propre.

l est donc manifeste, à travers tous ces exemples, que le pronom tu apparaît dans la bouche du locuteur en position haute.

\subsection{L'interpellation par un titre ou une fonction}

Le choix d'appellatifs qui font référence à une fonction, - qu'il s'agisse d'une fonction sociale (maître, esclave, époux...) ou familiale (mère, fils, sœur...) - n'est pas neutre. Ils rappellent les liens de pouvoir qui unissent les locuteurs entre eux ${ }^{20}$ et se rencontrent majoritairement dans des contextes où il $\mathrm{y}$ a un décalage de "place" entre les interlocuteurs (maître/esclave; épouse/mari; parent/enfant), que ce soit pour des raisons de catégorie sociale, de parenté, ou des raisons contextuelles. Dans ce cas, l'usage $\mathrm{du}$ titre permet, dans la bouche du personnage en position basse, de compenser un éventuel FTA commis contre son supérieur en montrant qu'il a conscience de leurs «places» respectives; dans celle du personnage en position haute, de conforter sa position d'autorité.

\subsubsection{Les esclaves à leurs maîtres}

Les esclaves emploient usuellement "ere » quand ils s'adressent à leur maitre. Par exemple en Ba $905^{21}$, l'esclave Chrysale adresse une demande à son maitre, ce qu'il n'est a priori pas en position de faire et il doit compenser ce FTA :

Ch: (...) Sine me - per te, ere, opsecro,

Deos immortalis - ire huc intro ad filium.

«Permets, mon maître, au nom des dieux immortels, que j'entre ici trouver ton

fils. » 


\section{les conjoints entre eux}

Entre époux, les apostrophes usuelles sont, de la part du mari, uxor (ou mea uxor), et de la femme, mi uir ${ }^{22}$. Chez Plaute, l'épouse emploie toujours un possessif quand elle s'adresse à son époux pour l'amadouer en marquant sa soumission et son affection ${ }^{23}$, car, de par son statut, elle est en position basse. C'est une façon subtile de se jouer des rapports de « place » imposés par l'ordre social. Ainsi, en Am 502-503, Alcmène essaie de retenir son mari qui part ${ }^{24}$ :

Alc : Quid istuc est, mi uir, negoti quod tu tam subito domo

Abeas? (...)

"Quelle affaire as-tu donc à mener, mon cher mari, pour partir si rapidement de la

maison?»

Concernant l'emploi du possessif, Joffre $(2007,127-129)^{25}$ précise à juste titre que quand l'appellatif est accompagné d'un adjectif possessif, cet adjectif est à rapprocher d'un datif de validation (pour preuve, on évoquera la forme de possessif de première personne, mi, datif d'un ancien *moi de pronom personnel de la première personne) : « On comprend la visée pragmatique de tels tours : non contents de solliciter l'attention de l'interlocuteur, ils contribuent également à la captatio beneuolentiae. Le locuteur cherche donc les bonnes grâces de son auditeur en lui signifiant que pour lui il est un être d'exception. De là proviennent la plupart des traductions affectives. »

La relation entre époux est a priori inégale puisque le mari a tout pouvoir sur sa femme, et c'est ce que ces apostrophes peuvent servir à manifester. Par exemple, en Cas $249^{26}$, Lysidame emploie l'expression uxor pour rappeler à sa femme qui est le maître :

Ly: Ohe, iam satis, uxor, es, comprime te, nimium tinnis.

"Hé, femme, maintenant ça suffit, calme toi ; tu fais trop de raffut. »

Plaute va exploiter ces appellatifs de fonction à des fins comiques, en soulignant par ce biais les renversements de «place». Ainsi, en As 926-927, Déménète, qui tente de calmer son épouse bafouée et très en colère, répète uxor, dans une vaine tentative pour lui rappeler que c'est lui qui a le pouvoir dans le couple. Malheureusement, il échoue lamentablement; il faut dire qu'il n'est, d'emblée, pas crédible, puisqu'il assortit cet appellatif de la supplication obsecro, qui neutralise la tonalité autoritaire qu'il tente de prendre $^{27}$ :

De : Iam obsecro, uxor, ....

Art : Nunc uxorem me esse meministi tuam?

Modo, cum dicta in me ingerebas, odium, non uxor, eram.

«-Je t'en prie, ma femme..

- Maintenant, tu te souviens que je suis ta femme? Tout à l'heure, quand tu

déblatérais contre moi, j'étais une horreur, non ta femme. »

\subsubsection{Parents et enfants ; frères et sœurs}

Entre parents et enfants, les parents appellent en général leur fils : gnate, ou mi gnate, leur fille filia (Men 844) (ou mea filia, comme en Rud 1173) ou encore (mea) gnata (Per 710), ce qui est pour eux un moyen subtil et efficace de rappeler leur fonction de parent et le respect qui leur est dû. Quand les enfants répondent en disant pater, ou mater, l'emploi de cet appellatif relève encore, dans la bouche d'un personnage en position basse, d'une volonté d'amadouer l'autre; dans ce cas, le terme d'adresse est souvent assorti d'un possessif visant à luidonner une coloration affectueuse. 

inconduite avec la fille d'Euclion et de révéler qu'il va avoir un enfant avec cette jeune fille. Il lui faut gagner la faveur de sa mère pour se faire pardonner cette incartade, et pour qu'elle l'autorise à épouser la jeune fille en question ${ }^{28}$ :

Lyc : (...) Nunc te obsecro

Resecroque mater, quod dudum obsecraueram:

Fac mentionem cum auonculo, mater mea.

«Maintenant, je t'en prie et t'en supplie, mère, comme je t'en avais priée tout à

l'heure : parles-en à mon oncle, ma chère mère. "

Lyconide va encore interpeller sa mère du titre mater mea aux vers 690 et 692, tandis qu'au vers 696, il emploie mater, soit au total 5 occurrences du terme en 13 vers.

En As 827-904, le vieillard Déménète est en train de banqueter aux côtés de Philénie, la maitresse de son fils Argyrippe ; puisqu'il a eu la gentillesse de donner à son fils l'argent nécessaire pour s'assurer les services de la belle toute une année, il considère que lui, son père, a le droit d'en profiter au moins une journée. Afin de faire passer cette pilule fort amère, il rappelle sans cesse son statut de père en appelant son fils gnate mi (3 occurrences). Argyrippe, obtempère en répétant pater (5 occurrences), peut-être avec la volonté de rappeler subtilement que l'attitude de Déménète est inconvenante pour un père.

De : Numquidnam tibi molestumst, gnate mi, si haec nunc mecum accubat?

$\mathrm{Ar}$ : Pietas, pater, oculis dolorem prohibet (...) (As. 830-831)

«- Est-ce que cela te gêne en quelque chose, mon fils, si cette femme est allongée à

ma table?

- La piété filiale, père, empêche mes yeux de souffrir. »

Au titre des liens familiaux figurent enfin les adresses par soror et frater. En Au 120-176 ${ }^{29}$, Eunomie veut convaincre son frère de se marier. Pour se faire pardonner cette ingérence, elle emploie le titre frater.

Au 120-122 ; 127-128: Eun : Velim te arbitrari med haec uerba, frater,

Meai fidei tuaique rei [haec]

Causa facere, ut aequum est germanam sororem.

(...) Verum hoc, frater, unum tamen cogitato,

Tibi proximam me mihique esse item te.

"Mon frère, je voudrais que tu comprennes que si je te tiens ce discours, c'est à cause de ma fidèle affection pour toi et dans ton intérêt, comme il convient que le fasse une sœur. (...). Mais, mon frère, pense bien à cela : je suis la personne la plus proche de toi, et vice versa. »

\subsection{L'interpellation par un substantif peu marqué comme homo, uir ou mulier}

Quand il faut interpeller un individu que l'on ne connaît pas, le locuteur recourt à des termes généraux, non marqués, tels homo, uir ou mulier, qui se contentent de faire référence au sexe de l'interlocuteur. Un adjectif possessif est souvent associé à ces formules de salutation, pour marquer l'amabilité et le respect ${ }^{30}$. En Cist 723, la servante Halisca qui a perdu la cassette de sa maîtresse demande de l'aide aux gens qu'elle rencontre. Elle aborde poliment Phanostrate et Lampadion pour leur demander s'ils n'ont rien vu:

Ha : $<0>$ mi homo et mea mulier, uos saluto.

« Mon cher Monsieur, ma chère Dame, je vous salue. » 
Le terme homo, fréquemment accompagné du possessif $m i$, peut par ailleurs être utilisé pour établir une connivence avec un interlocuteur mâle. Dans ce contexte, homo peut se traduire par «mon ami » $»^{31}$. Par exemple en Ba 1155a, Philoxène veut faire un aveu à son ami Nicobule ${ }^{32}$ :

$\mathrm{Ph}:$ Quid ais tu, homo?

Ni: Quid, me uis?

«- Dis-donc, compère.

- Qu'est-ce que tu veux?»

Cist 678-679, Halisca demande aux spectateurs s'ils ont vu la cassette qu'elle a perdue :

Ha : Mi homines, mi spectatores, facite indicium, siquis uidit

[si] quis eam abstulerit, quis[ue] sustulerit, et utrum hac an illac iter institerit.

«Mes amis, chers spectateurs, indiquez-moi si quelqu'un l'a vue, dites-moi si

quelqu'un l'a prise ou l'a enlevée, et s'il est parti par ici ou par là. »

\subsection{L'interpellation par une injure}

4

En français, employer une injure a pour but de blesser l'autre et celui qui l'emploie se met de fait en position haute, comme le souligne Kerbrat-Orecchioni $(1992,95)$. Il est probable qu'il en allait de même en latin, et c'est sa position haute qui autorise le maître à s'adresser usuellement en ces termes à son esclave.

Cependant, si l'injure est théoriquement un FTA, dans le cas de relations sociales aussi inégalitaires que celles qui unissent maitre et esclave, on peut se demander s'il en est encore ainsi. En effet, si l'injure devient un mode d'adresse banal, elle perd alors la valeur offensive liée à son caractère transgressif. Perret $(1968,13)$ soulignait bien, à propos du français, que si l'injure est un acte agressif, c'est parce qu'elle brise les normes sociales habituelles, en introduisant un lien intime entre des personnes qui ne le sont pas $^{33}$. Or, chez Plaute, le maître n'est pas tenu par la nécessité d'être poli vis-à-vis de l'esclave qui n'a aucune existence dans le réseau social. En outre, l'intrigue traditionnelle de la comédie ancienne veut que l'esclave ait berné son maître, qui a donc toutes les bonnes raisons du monde de l'injurier ${ }^{34}$ (injures qui sont généralement assorties de sanctions corporelles). L'injure ne serait donc dans la comédie ancienne que la marque de la supériorité sociale du maître ${ }^{35}$.

Par exemple, en Au 628-629 et Au 633, le vieil Euclion s'en prend à l'esclave Strobile qu'il soupçonne de l'avoir volé :

Euc : $<$ I $>$ foras, lumbrice, qui sub terras erepsisti modo,

Qui modo nusquam comparebas : nunc cum compares, peris. (...)

Verberabilissime, etiam rogitas, non fur, sed trifur?

« Sors de là, ver de terre, qui vient de ramper de sous le sol, qui ne te montrais nulle part tout à l'heure: maintenant puisque tu te montres, tu es mort. (...) Mégapendard, tu poses encore des questions. Non pas voleur, mais triple voleur !»

On a le sentiment qu'ici l'auteur comique s'amuse avec le public : la loi du genre veut que les maîtres insultent les esclaves; Plaute s'amuse en inventant les injures les plus extravagantes pour le plaisir des mots ${ }^{36}$. 
classe sociale, elles peuvent au contraire servir de marqueur de solidarité, comme quand elles sont utilisées entre esclaves. Comme le soulignent Lagorgette et Larrivée $(2004,83)$ : «certaines insultes ne visent pas à accomplir l'acte d'insulter mais bien au contraire servent à marquer la solidarité dans un groupe de pairs. » Dans le cas des esclaves, on a des personnages qui appartiennent à un même groupe social, où les relations de solidarité ont a priori besoin d'être fortes pour affronter le groupe des maîtres qui est en position de pouvoir absolu par rapport à eux ${ }^{37}$. L'échange d'injures paraît alors être un mode d'interaction spécifique par lequel les esclaves jouent ensemble ; c'est sans doute aussi une manière de se moquer des maîtres en leur empruntant les termes par lesquels ces derniers s'adressent à eux. Ainsi, en As. 297-298, deux esclaves, Léonide et Liban, se rencontrent, et, au lieu de se saluer par leur nom, ils échangent des injures, comme si celles-ci les définissaient mieux.

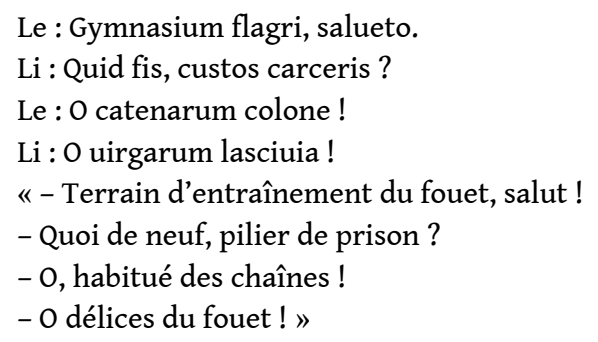

\subsection{L'interpellation par un terme affectueux ou flatteur}

Les amants de comédie échangent traditionnellement des mots doux qui vont rappeler les sentiments qui les unissent et renforcer le lien interlocutif. En Cur 164-165, on assiste ainsi à un échange entre Phédrome et Planésie :

$\mathrm{Ph}$ : Assum ; nam si absim, haud recusem quin mihi male sit, mel meum.

$\mathrm{Pl}$ : Anime mi, †procul amantem abesse haud consentaneumst.

«-Je suis là ; car si j'étais absente, je veux bien qu'un malheur m'arrive, mon doux miel.

- Mon cœur, il ne convient pas qu'un amant soit au loin.» 
Mais les mots doux ${ }^{38}$ peuvent aussi servir à séduire : le locuteur qui tente de séduire son interlocuteur dévoile ses réserves en montrant qu'il a besoin de lui; il prend un risque et se trouve de fait en position basse. En employant des appellatifs tendres, il va rechercher la faveur de l'interlocuteur pour renverser la situation et acquérir la position haute. En As 664, Philénie, qui tente de charmer les esclaves Léonide et Liban pour obtenir de l'argent, accumule ainsi les qualificatifs tendres ; mais Léonide ne se laisse pas séduire et conserve la haute main sur la conversation :

$\mathrm{Ph}: \mathrm{Da}$, meus ocellus, mea rosa, mi anime, mea uoluptas,

Leonida, argentum mihi ; ne nos diiunge amantis.

Le : Dic tigitur me tuum $†$ passerculum, gallinam, coturnicem,

Agnellum, haedillum me tuum dic esse, uel uitellum :

Prehende auriculis, compara labella cum labellis. (As. 664-668)

«-Mon cher œil, ma rose, mon cœur, ma joie, Léonide, donne-moi l'argent. Ne nous sépare pas, mon amant et moi.

- Appelle-moi donc ton petit moineau, ta poule, ta caille; dis que je suis ton petit agneau, ton petit chevreau ou ton petit veau. Prends-moi par les oreilles, place tes lèvres sur les miennes. "

Comme on le voit à partir de cette accumulation de termes, le substantif affectueux est fréquemment accompagné d'un possessif, ou affecté d'un diminutifi' ${ }^{39}$, qui va, tout comme le possessif, dans le sens de la captatio beneuolentiae.

51 Un appellatif aimable peut aussi être une façon pour le locuteur de manifester sa reconnaissance et sa joie à quelqu'un qui lui a rendu service ou lui a fait plaisir ${ }^{40}$, comme en Rud 1265-1266, où Pleusidippe s'adresse à son esclave Trachalion en ces termes :

$\mathrm{Pl}$ : Iterum mihi istaec omnia itera, mi anime, mi Trachalio,

Mi liberte, mi patrone potius, immo mi pater.

« Redis-moi tout cela mon cœur, mon cher Trachalion, mon affranchi, mon patron

plutôt, et même mon père. »

Enfin, quand c'est un maître qui s'adresse à son esclave avec une apostrophe aimable, c'est le signe qu'il se trouve en position basse vis-à-vis de lui et ce renversement de situation est d'autant plus comique que, comme nous l'avons dit, le maître utilise usuellement des injures à son égard. Par exemple, en As. 689, Argyrippe supplie ainsi son esclave Liban : o Libane, mi patrone («o Liban, mon patron $»)^{41}$.

\subsection{Conclusion}

53 En conclusion, les appellatifs de la comédie romaine visent à renforcer le lien conversationnel entre les interlocuteurs. Qu'ils se trouvent à l'appui d'une salutation, d'une demande, d'un ordre, d'un aveu, ils servent à attirer l'attention de l'autre et à l'impliquer dans la discussion. Dans ce but, ils peuvent être renforcés d'un adjectif possessif qui joue le rôle d'amadoueur. Il s'agit de manipuler l'autre par les mots pour faire avancer l'intrigue. En ce sens, ces différents appellatifs visent à renforcer la position $\mathrm{du}$ locuteur qui prend à sa charge le bon déroulement de l'échange et pousse l'interlocuteur à entrer dans son jeu.

Plus spécifiquement, l'usage d'un titre, d'un terme rappelant les liens sociaux ou familiaux qui unissent les interlocuteurs permet de renforcer la position haute de celui qui l'emploie, ou au contraire, quand il est employé par un locuteur en position basse, de compenser un FTA. 
De façon générale, le fonctionnement des appellatifs dans la comédie ancienne n'est pas différent ce celui qu'on relève dans les interactions quotidiennes en français contemporain (mis à part le cas particulier des rapports maitre / esclave qui est une donnée spécifique de l'époque antique).

\section{Les interpellations dans les tragédies de Sénèque}

Après cet aperçu des emplois de l'interpellation dans les comédies de Plaute, tournonsnous vers le corpus des tragédies de Sénèque afin de présenter de manière contrastive le fonctionnement des appellatifs dans ces deux genres littéraires.

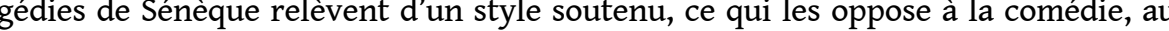
style familier et, pour autant qu'on puisse en juger, proche de la langue parlée. Les tragédies antiques se caractérisent par ailleurs par leurs fréquents monologues, dans lesquels le héros tragique chante sa souffrance ${ }^{42}$. Nous ne nous intéresserons pas ici aux apostrophes qui constellent les monologues, mais nous focaliserons notre analyse sur les interpellations « authentiques ", dans un dialogue adressé à un autre personnage, présent sur scène.

\subsection{Les contextes d'emploi des appellatifs}

$58 \mathrm{Au}$ titre des points communs avec la comédie, on relèvera les contextes dans lesquels apparaissent les appellatifs.

\subsubsection{Les débuts de dialogue}

Comme dans les comédies, les termes d'adresse sont nombreux dans les débuts de dialogue : ils permettent d'accueillir l'autre dans la conversation, tout en l'identifiant pour le spectateur ${ }^{43}$. Mais, à la différence de la comédie, dans les tragédies, l'entrée en dialogue se fait de façon abrupte: on apostrophe l'interlocuteur et entre directement dans la conversation par le biais d'une question ou d'un ordre qui incitent tout de suite l'autre à parler ou à agir. Nommer l'interlocuteur permet de lui reconnaître d'emblée le statut d'interlocuteur et c'est peut-être une des raisons pour lesquelles Sénèque peut se permettre de faire l'économie des formules de salutation qui étaient si fréquentes dans la comédie ${ }^{44}$.

On évoquera à titre d'exemple le passage suivant où, après avoir monologué sur quatre vers, ๔edipe accueille Créon en l'interpellant par un titre assez développé, en guise de salutations :

CEd 210-211 : Oed : Germane nostrae coniugis, fessis opem

Si quam reportas, uoce properata edoce.

«Frère de mon épouse, si tu apportes une aide quelconque aux malheureux que

nous sommes, dis-le nous rapidement. "

61 Créon n'est pas choqué par cette abrupte entrée en matière puisqu'il va directement répondre à la question qui lui est implicitement posée, sans prendre non plus le temps de saluer CEdipe.

Cette façon de situer le personnage par rapport à sa parentèle est typique d'un contexte mythologique ${ }^{45}$; on en trouve un autre exemple en HF 309, où Amphitryon tente d'entamer une conversation avec Mégare qui monologuait jusqu'alors; pour attirer son 
attention, il l'interpelle en ces termes : o socia nostri sanguinis ${ }^{46}$. La formule joue le rôle de «summons ».

\subsection{2 À l'appui d'un ordre, d'une question ou d'une demande insistante}

63 Comme dans la comédie, l'appellatif peut aussi avoir une fonction conative ; il apparaît en soutien d'un ordre ou d'une demande ${ }^{47}$, pour rappeler à l'interlocuteur qu'il est partie prenante de l'échange et donc l'inciter à prêter attention à ce qui lui est dit.

En Med 568, Médée, furieuse parce que Jason la chasse, monologue et s'interrompt pour donner un ordre à la nourrice ${ }^{48}$ :

Med : Tu, fida nutrix, socia maeroris mei

Variique casus, misera consilia adiuua.

«Toi, fidèle nourrice, compagne de mon chagrin et de mes divers malheurs, viens

en aide à mes tristes projets. »

65 De la même façon, le terme d'adresse peut venir en appui à une question. En Agam 953-954, Clytemnestre entre en scène et interpelle Electre qui s'y trouve déjà :

Cly : Hostis parentis, impium atque audax caput,

Quo more coetus publicos uirgo petis?

«Toi l'ennemie de ma mère, être impie et audacieux, comment une vierge comme

toi peut-elle se montrer en public?»

L'apostrophe permet ici encore de passer l'étape des salutations, pour entrer dans la conversation de manière assez abrupte par une question, qui lui laisse à l'autre peu de latitude pour refuser l'échange. Pas de «small talk » ici. La tragédie fait l'économie des modes d'interaction polis dont on trouvait des traces dans la comédie, qui comportait des séquences d'ouverture de la conversation assez développées.

Quand un personnage en position basse est amené à donner un ordre à quelqu'un qui lui est supérieur dans la relation énonciative, le choix du bon appellatif est particulièrement important. Utiliser un terme comme era permet à une servante de manifester à sa maîtresse le respect qui lui est dû, ce qui est particulièrement nécessaire s'il faut compenser un « FTA ». Ainsi, en Med 425-426, la nourrice donne à sa maîtresse un conseil qui pourrait être mal interprété au vu de sa position; pour atténuer ce qui pourrait être perçu comme une insolence, elle emploie l'apostrophe era :

Nut : Recipe turbatum malis

Era, pectus, animum mitiga.

«Maîtresse, reprends tes esprits perturbés par les malheurs, et calme ta colère. »

En HO 275-276, la nourrice est encore plus subtile : au lieu de manifester le respect dû à sa maîtresse, elle joue sur l'affectif en rappelant à celle-ci qu'elle l'a nourrie, puisqu'elle l'appelle alumna, littéralement «nourissonne » pour reprendre le néologisme de Dupont $(1995,110)$ :

Nut : Pectoris sani parum

Aestus, alumna, comprime et flammas doma.

«Mon enfant, calme le feu de ton cœur furieux, et dompte ses flammes. »

69 Au-delà de la fonction de renforcement du lien interlocutif, le choix de l'appellatif ne se fait donc pas au hasard. Selon l'aspect de l'interlocuteur qu'on choisit de mettre en valeur, on va le rendre plus réceptif au discours. 


\subsection{3 À l'appui d'un aveu ou d'une confidence}

Comme dans la comédie, les apostrophes sont fréquentes lors d'un aveu ou d'une confidence pour susciter une écoute attentive de la part de l'interlocuteur. Par exemple, en Thy 512-514, Thyeste, très ému, avoue à son frère Atrée ses fautes passées ${ }^{49}$ :

Thy : Sed fateor, Atreu, fateor, admisi omnia

Quae credidisti.

«Mais je l'avoue, Atrée, je l'avoue, j'ai commis tous les actes que tu as crus.»

\subsubsection{Pour marquer un changement d'interlocuteur}

71 Au-delà de ces emplois de l'interpellation visant à souligner le lien interlocutif, on relève aussi, comme chez Plaute, de simples emplois fonctionnels où l'apostrophe vise à indiquer à qui l'on parle dans le cas d'un polylogue. L'appellatif va alors renforcer ce qui était indiqué par l'attitude et les regards du locuteur. Par exemple, en Agam 932-94350, Strophius parle à tour de rôle à Oreste, à son fils Pylade, et à ses chevaux. Par souci de clarté, sans doute, Sénèque fait figurer à chaque fois un appellatif pour indiquer quel est l'interlocuteur visé :

\section{ST : Etsi timendum caesus Agamemnon docet,}

Aggrediar et te, Oresta, furabor libens. (...)

Tuque, o paternis assidens frenis comes,

Condisce, Pylade, patris exemplo fidem.

Vos Graecia nunc teste ueloces equi,

Infida cursu fugite praecipiti loca.

«Bien que le meurtre d'Agamemnon me prévienne qu'il faut avoir peur, je m'avancerai et toi, Oreste, je te cacherai volontiers. (...) Et toi, qui t'assieds en compagnon, près des rênes de ton père, apprends, Pylade, par l'exemple de ton père, ce qu'est la fidélité. Et vous, chevaux rapides (la Grèce peut en témoigner), fuyez ces lieux de trahison dans une course précipitée. »

Comme nous avons pu le voir à travers les exemples précédents, les appellatifs sont aussi très fréquents à l'issue d'un monologue pour indiquer au public que le locuteur cesse de se parler à lui-même pour se tourner vers autrui et entrer dans l'interaction. Ce type d'emploi était fréquent en comédie, mais est sans doute plus indispensable encore dans les tragédies où les monologues abondent. On évoquera à titre d'exemple les passages de Med 568, ou Med 974 où Médée quitte le monologue pour parler à sa nourrice en l'appelant fida nutrix, ou comes.

73 Les appellatifs s'emploient donc dans des contextes similaires dans le corpus comique et le corpus tragique ; en revanche, si l'on se penche sur la nature des appellatifs utilisés, on est frappé par les différences d'emploi.

\subsection{La nature des appellatifs}

Concernant la nature des termes utilisés, les différences sont grandes entre les deux genres littéraires. Dans la tragédie, les noms propres sont beaucoup moins employés que dans la comédie. 


\subsubsection{Les noms propres} entre eux d'appellatifs qui sont principalement des titres soulignant leur fonction, ou indiquant le lien familial ${ }^{51}$ qui les unit. Nous pouvons évoquer le passage de Agam 910, 914, 917, où, sans doute par un souci de uariatio, Electre appelle son frère de trois manières différentes :

$\mathrm{El}$ : Fuge, o paternae mortis auxilium unicum, (...)

Germane, uultus ueste furabor tuos.

(....) Pone iam trepidos metus,

Oresta : amici fida praesidia intuor.

«Fuis, o seul recours à la mort de notre père. (...) Mon frère, je cacherai de mon vêtement ton visage. Renonce à tes peurs qui te font trembler, Oreste : je vois ici un ami qui nous protègera fidèlement. "

Les apostrophes peuvent être particulièrement développées quand Sénèque greffe une apposition ou une relative déterminative sur le terme au vocatif. Ces expansions de l'appellatif vont apporter des précisions supplémentaires sur le personnage visé et permettre au public de mieux cerner les enjeux de la situation d'énonciation ${ }^{52}$. Par exemple, en Tro 353-359, Agamemnon accueille le devin Calchas avec des titres ronflants ; il s'agit de dégager le pouvoir et la dignité de Calchas, qui font qu'on ne peut que l'écouter et se plier à ses avis :

Aga : Tu qui Pelasgae uincla soluisi rati Morasque bellis arte, qui reseras polum, Cui uiscerum secreta, cui mundi fragor

Et stella longa semitam flamma trahens

Dat signa fati, cuius ingenti mihi

Mercede constant ora, quid iubeat deus

Effare, Calchas nosque consilio rege.

« Toi qui a su défaire les liens de la flotte des Pélasges et les retards de la guerre, toi qui, par ton art, dévoile le ciel, à qui les secrets des viscères, le fracas du monde et 
l'étoile traçant son chemin d'une longue flamme indiquent les signes du destin, toi dont les paroles me coûtent si cher, dis-moi, Calchas, ce qu'ordonne la divinité et conduis-nous par tes conseils. »

Pour expliquer cette prédominance des titres en apostrophe, nous pouvons invoquer plusieurs explications: tout d'abord, la convention extrême qui prévaut dans les dialogues tragiques : chaque personnage est prisonnier de sa fonction qui va déterminer le type de discours qu'il est en droit de tenir ${ }^{53}$, mais aussi celui qu'il est en droit d'entendre de la part de son interlocuteur.

En particulier, dans les échanges où le locuteur incarne la société humaine et tente de ramener le furieux à la raison (typiquement, dans les dialogues nourrices / maitresses ${ }^{54}$ ), l'usage d'un titre pour interpeller le furieux vise à lui rappeler ce qu'il est, et les obligations sociales qui en découlent. Par exemple, en Phèdre 129-130, la nourrice interpelle Phèdre avec la plus grande solennité pour lui rappeler ses obligations sociales :

Nut : Thesea coniunx, clara progenies Iouis,

Nefanda casto pectore exturba ocius.

« Femme de Thésée, célèbre descendante de Jupiter, chasse vite de ton chaste cœur ces sentiments impies. »

81 En Phèdre 864-865, c'est Thésée qui essaie de raisonner une Phèdre suicidaire :

Th: O socia thalami, sicine aduentum uiri

Et expetiti coniugis uultum excipis?

« $\mathrm{O}$, compagne de ma couche, est-ce ainsi que tu accueilles l'arrivée ton mari et la vue de ton époux que tu désirais?»

L'usage du titre dans les apostrophes permet en outre de souligner a contrario en quoi le furieux transgresse ces institutions sociales. Quand une belle-mère aime son beau-fils et n'hésite pas à braver les interdits sociaux, c'est un acte abominable, et le terme de mater, qu'Hippolyte applique à Phèdre, vise précisément à souligner par contraste ce que la passion de celle-ci a de scandaleux ${ }^{55}$ :

Hip : Committe curas auribus, mater, meis.

$\mathrm{Ph}$ : Matris superbum est nomen et nimium potens :

Nostros humilius nomen affectus decet ;

Me uel sororem, Hippolyte, uel famulam uoca,

Famulamque potius. (...) (Phèdre 608-611)

«- Confie moi tes soucis, ma mère.

- Le nom de mère est trop solennel et trop puissant. Un nom plus humble convient

à mon affection. Appelle-moi ta sœur, ou ton esclave, Hippolyte; ton esclave

plutôt. »

3 Notons par ailleurs que, dans ce passage, Phèdre appelle uniquement Hippolyte par son nom, probablement pour éviter de lui donner un titre qui rappellerait leur lien familial (v. $611,646,710)$. Le terme utilisé en apostrophe concentre donc l'essence de la tragédie. On en a encore deux exemples particulièrement cruels en Med 1024 et Thy 1004 où Médée et Atrée interpellent respectivement Jason et Thyeste $\mathrm{du}$ nom de parens, alors qu'ils viennent de tuer les enfants de ces hommes.

Enfin, il nous faut invoquer des raisons stylistiques à cette prédominance des titres dans les interpellations tragiques. Pour Dickey $(2002,32)$, plus le style est élevé, plus le terme d'adresse est développé : "There are certain register-dependent stylistic traits of Latin addresses which seem to function independently (for the most part) of the particular words used. The simplest such distinction is that complex addresses (those made up of two or more words, not counting the particle o) are most likely to be found in texts exhibiting a more literary style, while those registers closer to conversational language 
have a stronger preference for one-word addresses. Thus, in the tragedies of Seneca, which contain perhaps the most literary language of any text considered for this study, $29 \%$ of the addresses consist of more than one word. »

\subsubsection{Le pronom de deuxième personne}

Comme chez Plaute, le pronom de deuxième personne sert aussi à l'interpellation. Comme dans la comédie, ce type d'apostrophe se rencontre dans la bouche de personnages en position haute, et il est très souvent développé par une apposition. Nous renverrons ici, à titre d'exemple, aux passages d'Agam 932-943 et Med 568, cités ci-dessus.

\subsubsection{Les injures}

Il nous reste à évoquer le cas particulier des injures, beaucoup plus rares dans la tragédie que dans la comédie, ce qui s'explique aisément par la différence de registre entre ces deux genres littéraires ${ }^{56}$. Quand on rencontre des injures, elles sont donc bien moins truculentes que celles de Plaute, qui font souvent allusion à l'esclavage ou aux animaux. Comme le fait remarquer Dickey $(2002,177)$, il n'y a aucune vulgarité dans le vocabulaire tragique.

De nombreuses injures, peu agressives, tournent autour de la perte de raison; ainsi des termes comme amens (cf. Agam 244, d'Egisthe à Clytemnestre), demens (Agam 960 de Clytemnestre à sa fille), furibunda (Agam 981, d'Egisthe à Electre : furibunda uirgo), furiosa (Agam 1011, de Clytemnestre à Cassandre). Le but est de réprimander l'autre pour le faire réagir. Mais les termes utilisés peuvent être plus violents ; ainsi, en Phèdre 687-689, Hippolyte, horrifié par la déclaration de Phèdre, lui répond avec indignation :

Hip : O scelere uincens omne femineum genus,

O maius ausa matre monstrifera malum,

Genetrice peior. (...)

«O toi qui dépasses en crime toute la race des femmes, qui as osé un forfait plus grand que celui de ta mère qui as porté un monstre en ses flancs, tu es pire que ta mère. »

Ou encore, en Tro $750-756^{57}$, Andromaque injurie Ulysse qui lui annonce qu'il va tuer son enfant :

And : O machinator fraudis et scelerum artifex,

Uirtute cuius bellica nemo occidit,

Dolis et astu maleficae mentis iacent

Etiam Pelasgi, uatem et insontes deos

Praetendis? Hoc est pectoris facinus tui.

Nocturne miles, fortis in pueri necem,

Iam solus audes aliquid et claro die.

«Toi le machinateur de tromperies et l'artisan de crimes, qui n'a jamais tué personne par ton courage à la guerre, mais qui a anéanti même les Pélasges par les ruses et l'astuce de ton esprit pervers, tu prends comme prétexte le devin et les dieux innocents? Ce crime vient de ton cœur. Soldat nocturne, courageux quand il s'agit de tuer un enfant, tu oses enfin faire quelque chose seul et en plein jour ! » lâche ${ }^{58}$. 


\subsubsection{Les termes affectueux}

Enfin, rares sont les termes affectueux dans les tragédies, pour la simple raison que, par définition, il n'y a pas d'amour tragique heureux, et donc pas de raison d'y échanger des roucoulades. Les seuls termes tendres se rencontrent dans les échanges entre parents et enfants mais ils restent rares car la tragédie n'est pas le lieu de l'épanchement de sentiments du quotidien. Nous pouvons cependant évoquer le passage de Tro 766-767, où Andromaque adresse des mots d'amour maternel ${ }^{59}$ à Astyanax, son fils qui va mourir :

And : $O$ dulce pignus, o decus lapsae domus,

Summumque Troae funus, o Danaum timor (...).

" O toi, mon doux gage d'amour, ô toi, gloire d'une maison anéantie, dernière

victime de Troie, terreur des Danaens. »

On peut aussi faire rentrer dans la catégorie des termes affectueux les mots altrix (Phaedr 251 ; HO 396) et alumna (Phaedr 255, ou HO 276) qu'échangent les héroïnes de tragédie avec leurs nourrices et qui rappellent ce lien fort et ancien qui les unit.

\subsection{Conclusion}

Dans la tragédie, les jeux sont faits d'avance puisque c'est une histoire mythique qui est mise en scène, dont le public connaît d'emblée l'issue ${ }^{60}$. Pour reprendre les termes de Dupont $(1985,193)$, «Ce que nous appelons action n'existe pas dans la tragédie romaine, et le suspense encore moins. » Les apostrophes n'ont donc pas le rôle clef qu'elles peuvent prendre dans la comédie où l'esclave meneur de l'intrigue doit manipuler les autres personnages pour parvenir à ses fins, les convaincre, les séduire, ou les provoquer.

C'est sans doute précisément parce que chacun est enfermé dans son rôle et que la tragédie revendique implicitement ses propres codes esthétiques, que les longues apostrophes qui définissent les fonctions de chacun (parfois sur deux vers) y sont si fréquentes. Comme l'explique Dupont $(1985,190)$, dans la tragédie, «chaque héros a un rôle donné d'avance, antérieur à la tragédie elle-même, rôle qui ne peut pas être modifié ». Le plaisir du spectateur passe par la reconnaissance de ces personnages; les appellatifs ont un rôle clef à jouer dans cette perspective. Les choses sont bien différentes dans la comédie où le public sait que le meneur de jeu va s'en sortir et résoudre le problème qui lui est soumis, mais où le suspense repose précisément dans la manière par laquelle il va y parvenir.

\section{Conclusion}

4 En conclusion, si l'on tente de dégager les points communs entre comédie et tragédie, on relèvera des similitudes dans l'usage des appellatifs :

* Vis-à- vis du public : aider le public à comprendre quels sont les personnages sur scène est quelle est la relation qui les unit, en particulier sur l'axe vertical ${ }^{61}$. Dans le cas particulier où il y a plusieurs personnages sur scène, les appellatifs servent aussi à indiquer qui est le destinataire de la réplique.

* En termes de dynamique du dialogue, - au-delà d'une fonction strictement vocative de l'appellatif en début de dialogue -, maintenir et renforcer le lien conversationnel, en 
impliquant davantage l'adressé dans l'échange, en particulier dans le cas d'un ordre ou d'une question (valeur conative), ou de l'expression de sentiments intimes.

Les injures et les mots tendres, qui traduisent les affects unissant les locuteurs, sont moins faciles à décrire en termes de "place». L'injure (sauf quand elle sert à marquer une solidarité de classe) permet au locuteur de se placer en position haute, en diminuant l'autre par le biais de ce «FTA ». Les mots affectueux constituent un «FFA » et sont le signe que le locuteur accepte une position basse, sauf dans le cas d'une tentative de séduction, où il s'agit au final de prendre l'ascendant sur l'autre.

Au chapitre des différences, on mettra en exergue la prédominance des titres pour interpeller autrui dans la tragédie, choix qui s'explique notamment par une volonté de plus grande solennité et par une écriture extrêmement codifiée.

La comédie à l'inverse fonctionne avec les modalités du dialogue quotidien et use des appellatifs d'une manière qui évoque nos usages contemporains. Cela s'explique par le fait que, pour avancer, l'intrigue dépend de l'interaction entre personnages, de la manière dont les uns vont manipuler les autres, ce qui passe notamment par le choix du bon appellatif.

\section{BIBLIOGRAPHIE}

Biville, F. (1998). Le statut linguistique des noms propres en latin. Approche formelle. In B.

García-Hernández (Ed.), Estudios de lingüística latina (t.2, pp. 825-839). Madrid : Ediciones clásicas.

Boyle, A.J. (1997). Tragic Seneca ; an essay in the theatrical tradition. London : Routledge.

Braun, F (1988). Terms of Address. Problems of patterns and usage in various languages and cultures.

Amsterdam : Mouton.

Brown, P. \& Levinson, S.C. (1987). Politeness. Some universals in language usage. Cambridge : CUP.

Détrie, C. (2006). De la non personne à la personne : l'apostrophe nominale. Paris : Éditions du CNRS.

Dickey, E. (2002). Latin Forms of Address. From Plautus to Apuleius. Oxford : University Press.

Ducrot,O. (1972). Dire et ne pas dire. Paris : Hermann.

Dupont, F. (1985). L'acteur-roi. Paris : Les Belles Lettres.

Dupont, F. (1995). Les monstres de Sénèque. Paris : Belin .

Dupont, F. $\left(1999^{2}\right)$. Le théâtre latin. Paris : Armand Colin.

Dupont, F. (2000). Médée de Sénèque, ou Comment sortir de l'humanité. Paris : Belin .

Féral, J. (1988). La théâtralité. Poétique, 75, pp. 347-361.

Gaide, F. (2001). A propos des interactions verbales dans le théâtre de Plaute . In C. Moussy (éd.), De lingua latina nouae questiones (pp. 959-969). Paris : Peeters. 
Joffre, M. D. (1998). Les possessifs de personne 1 et 2 : un moyen de faire surgir dans l'énoncé l'un des acteurs de l'énonciation. In B. Garcia Hernandes (Ed.), Estudios de linguistica latina (t. 1, pp. 409-417). Madrid : Ediciones Clasicas.

Joffre, M. D. (2007). Ocelle mi (Plaute, Trin 245), un emploi hors structure. In C. Bodelot (éd.), Eléments asyntaxiques ou hors structure dans l'énoncé latin (pp. 123-135). Clermont Ferrand : Presses Univ. Blaise Pascal.

Hoffmann, M. E. (1983). Conversation openings in the comedies of Plautus. In H. Pinkster (Ed.), Latin linguistics and linguistic theory (pp. 217-226). Amsterdam : J. Benjamins.

Kerbrat-Orecchioni, C. (1984). Pour une approche pragmatique du dialogue théâtral. Pratiques , 41, pp. 46-62.

Kerbrat-Orecchioni, C. (1988). La notion de « place » interactionnelle ou les taxèmes. Qu'est-ce que c'est que ça ?. In J. Cosnier, N. Gelas \& C. Kerbrat-Orecchioni, (éds.), Échanges sur la conversation (pp. 185-198). Paris : Éditions du CNRS,.

Kerbrat-Orecchioni, C. (1990). Les interactions verbales, t. 1. Paris : Armand Colin.

Kerbrat-Orecchioni, C. (1992). Les interactions verbales, t. 2. Paris : Armand Colin.

Kerbrat-Orecchioni, C. (2000). Est-il bon, est-il méchant : quelle représentation de l'homme-ensociété ?. In M. Wauthion \& A.C. Simon (Eds.), Politesse et idéologie. Rencontres de pragmatique et de rhétorique conversationnelles, (pp. 21-35). Louvain : Peeters.

Laver, J. (1981). Linguistic routines and politeness in greeting and parting. In F. Coulmas (Ed.), Conversational routine (pp. 289-304). The Hague : Mouton.

Levinson, S.C. (1983). Pragmatics. Cambridge : Cambridge University Press.

Lagorgette, D. \& Larrivée, P. (2004). Interprétation des insultes et relations de solidarité. Langue française, 144, pp. 83-103.

Lilja, S. (1965). Terms of abuse in Roman comedy. Helsinki : Annales Academiae Scientiarum Fennicae.

Mellet, S. \& Rollinat-Levasseur, E. M. (1989). Sénèque, Phèdre : Remarques à propos de l'expression de la personne, Vita Latina, 116, p. 37-42.

Müller, R. (1997). Sprechen und Sprache. Dialoglinguistische Studien zu Terenz. Heidelberg : Universitätsverlag C. Winter.

Perret, D. (1968). Termes d'adresse et injures ; à propos d'un dictionnaire des injures. Cahiers de lexicologie, 12, pp. 1-14.

Risselada, R. (1993). Imperative and other directive expressions in Latin : a study in the pragmatics of a dead language. Amsterdam : Gieben.

Roesch, S. (2007). Confidences, monologues et apartés dans les comédies de Plaute. In C. KerbratOrecchioni \& V. Traverso (éds.), Confidence/confiding, Dévoilement de soi dans l'interaction / Self disclosure in Interaction (pp. 59-81). Tübingen : Max Niemeyer.

Roesch, S. (2008). Les débuts de dialogue dans la comédie et la tragédie latines. In B. Bureau \& C. Nicolas (éds.), Commencer et finir ; débuts et fins dans les littératures grecque, latine et néolatine (t.1, pp. 207-222). Paris : de Boccard.

Serbat, G. (1987). Sur le vocatif. Vita Latina, 106, pp. 7-13.

Serbat, G. (1996). Grammaire fondamentale du latin, tome VI : l'emploi des cas en latin classique :

Nominatif, vocatif, accusatif, génitif, datif. Louvain : Peeters. 
Shalev, D. (1998). Vocatives in responses : a bridging mechanism in dialogue exchange ?. In B. García-Hernández (Ed.), Estudios de lingüística Latina (t.2, pp. 765-779). Madrid : Ediciones clásicas.

Schegloff, E.A. \& Sacks, H. (1973). Opening up Closings. Semiotica, 8, pp. 289-327.

Vairel, H. (1981). The vocative in the Latin case system. American Journal of Philology, 102, pp. 438-447.

Vairel, H. (1986). Le problème du cas de tu : Nominatiuus tantum, Vocatiuus tantum, ou Vterque. RPh, 60, pp. 31-57.

\section{NOTES}

1. Notre corpus de référence sera ici constitué des comédies de Plaute et des tragédies de Sénèque.

2. Voir aussi Thy 192, 324, 423.

3. Voir aussi HO 964, 972, 977, 982.

4. Cf. C. Kerbrat-Orecchioni (1988).

5. Sur le lien entre les valeurs du vocatif et le contexte pragmatique, voir Serbat $(1987,9)$.

6. Sur la répartition entre nominatif et vocatif, voir Serbat $(1996,106-107)$; Vairel $(1981,446)$.

7. Cf. Dupont (1999, 118-130).

8. Cf. aussi Perret $(1968,13)$.

9. Voir Hoffmann (1983) pour les séquences « summons-answer » dans la comédie.

10. Voir aussi Ba 456-457, 626.

11. Sur ce point, cf. Perret $(1968,6)$ : « lorsque j'énonce un nom propre au vocatif, j'affirme : vous êtes un être humain, je connais votre nom, je vous connais, ce nom est le vôtre.»

12. Compris comme «terme par [lequel] on s'adresse à quelqu'un » (Perret, 1968, 1).

13. Cf. Risselada $(1993,89)$.

14. Cf aussi par exemple Ba 493 ; Capt 319-320.

15. Pour d'autres exemples, voir Roesch (2007).

16. Voir aussi Ba 560, 683-685.

17. Sur le cas du pronom tu, comparer les positions de Vairel (1986) et Serbat (1996, 95-98).

18. Voir aussi As 446.

19. Voir aussi Cas 963 avec une séquence analogue : ubi es tu qui...

20. Cf. Joffre $(2007,128)$ sur ces termes.

21. Voir aussi As 641-643; Au 820-826.

22. Les épouses peuvent aussi s'adresser à leur époux en employant le prénom de celui-ci (cf. par exemple Am 540).

23. Le possessif permet peut-être aussi de désambiguïser uir qui est polysémique et ne signifie pas toujours « mari »; Müller faisait une remarque analogue $(1997,282)$ à propos de homo.

24. Voir aussi Am 716.

25. Cf. Détrie $(2006,56)$ et Joffre $(1998,416)$.

26. Voir aussi Am 521.

27. Cf. Détrie $(2006,57)$ pour un exemple, tiré de Balzac, de dissensus sur l'apostrophe entre les coénonciateurs. Chez Plaute, voir aussi Am 810-811, où Amphitryon interdit à sa femme de l'appeler uir, puisqu'il est persuadé qu'elle l'a trompé.

28. Voir As 511-544 : Philénie répète à 5 reprises le terme mater pour apaiser sa mère en colère.

29. Voir aussi Curc 641, 657 et 658.

30. Cf. le français Mon-sieur, Ma-dame.

31. Cf. Dickey (2002 : 222). 
32. Voir aussi Cist 719 ; Per 620 et Epid 640.

33. Cf. Perret $(1968,13)$. Sur cette question de l'effet injurieux de l'introduction de l'intime dans la sphère publique, voir Lagorgette et Larrivée (2004, 94-95).

34. Pour une description détaillée des termes d'injure chez Plaute (et Térence), voir Lilja (1965) ; pour Térence, voir Müller (1997, 266-280).

35. Voir aussi par exemple Am. 578-579 pour les termes carnufex et uerbero («bourreau, pendard») ou Am 565-572.

36. Cf. Lilja $(1965,82)$. Pour un jeu sur les injures, voir Capt 659-662.

37. Sur ce point et l'échange d'injures entre esclaves, voir aussi Gaide $(2001,961)$.

38. Pour une liste des appellatifs affectueux du latin, voir Dickey (2002, 152-154).

39. Pour les appellatifs affectueux chez Plaute, voir Gaide $(2001,964)$.

40. Voir aussi Ba 879.

41. Pour des exemples analogues, voir par exemple Cas 725, 738-739.

42. Sur le monologue tragique, voir Dupont (1985, 190-192).

43. Sur ce point, voir notre article (Roesch, 2008, 215).

44. Sur l'intérêt, dans la tragédie, d'identifier correctement le statut de la personne à laquelle on s'adresse, pour que l'échange s'effectue dans de bonnes conditions, nous renvoyons au passage d' EEdipe 1004 sequ., que nous avons déjà étudié ailleurs (Roesch, 2008, 215).

45. Cf. Dickey $(2002,113)$.

46. Voir aussi Agam 125.

47. Voir Tro 691-693.

48. Voir aussi par exemple Agam 910 (cité ci-dessous) ; HF 1185.

49. Voir aussi Med 246 ; HO 491.

50. Cf. aussi Agam 226-233; HO 99 et 101, ou 570 et 581.

51. Pour un classement des différents appellatifs familiaux, voir Dickey $(2002,112)$.

52. Voir aussi Med 266-269 ; HO 535, 569-570.

53. Cf. F. Dupont $(1995,108)$ : «Tout dialogue, tout débat verbal, n'est pas un duel, il y a bien d'autres modèles interlocutoires, on peut citer, outre la consolation, le conseil, consilium, la supplication, supplicatio, le revendication, petitio. (...) C'est pourquoi, au début d'un dialogue, les interlocuteurs s'apostrophent en s'adressant la parole afin de préciser le mode de rapport qu'ils entendent avoir l'un avec l'autre et qui commandera le contenu de leurs paroles.»

54. Cf. Dupont $(1995,109)$.

55. Voir aussi Thy 521, 530, 535, où le fait que Thyeste appelle de manière répétée Atrée frater souligne l'horreur de la trahison de ce dernier.

56. Dickey $(2000,173)$ dresse une liste des principales insultes du latin, en les classant suivant leur registre.

57. Voir aussi Tro 301-303.

58. Pour une intention analogue, voir Tro 1002, où Hécube interpelle Pyrrhus en le traitant mactator senum (« assassin de vieillards »).

59. Voir aussi Med 945.

60. Le personnage tragique lui aussi d'ailleurs, puisqu'il se sait prisonnier de son destin (cf. Med 910 : Medea nunc sum : « Maintenant, je suis Médée »)

61. Pour cette opposition axe horizontal / axe vertical, voir Kerbrat (1992, 39 sequ). 


\section{RÉSUMÉS}

Les interpellations entre personnages sont fréquentes dans les comédies comme dans les tragédies latines. De manière générale, comme il s'agit d'un corpus théâtral, les termes d'adresse servent à indiquer au public qui sont les personnages en scène et quels sont les rapports qui les unissent. Suivant le genre littéraire considéré, l'interpellation a cependant une fonction différente. Dans la comédie, les appellatifs contribuent à faire avancer l'action, en agissant sur l'interlocuteur de différentes manières : dès le début de l'interaction, ils permettent d'indiquer clairement et économiquement à l'interlocuteur quelle est la place que le locuteur revendique sur l'axe vertical (les noms de titres ou de fonctions sont particulièrement efficaces dans cette perspective). Stratégiquement, les appellatifs peuvent en outre servir à compenser un FTA ou à soutenir un FFA. Enfin, ils ont une fonction phatique de renforcement du lien communicationnel: ils permettent à la fois de soutenir l'attention de l'autre, mais aussi de l'accueillir dans le dialogue en le reconnaissant. Le niveau de langue peu soutenu de ce genre littéraire fait par ailleurs la part belle aux interpellations les moins marquées, comme le nom propre, ou le pronom de la deuxième personne. Les personnages tragiques à l'inverse n'ont pas à faire progresser une action qui est écrite d'avance. C'est en effet un mythe au déroulement inéluctable qui est représenté sur scène. Quand les personnages s'interpellent les uns les autres, ils le font dans des termes solennels, à l'aide d'un titre ou d'un terme qui indique la fonction de l'interlocuteur, ou à travers des périphrases qui le situent dans son histoire familiale.

In ancient Latin comedies and tragedies, the characters frequently address each other using vocatives. As these texts are meant for the stage, the address forms aim first at helping the audience to identify the characters speaking and understanding the relations of power between them. Depending on the literary genre, they have different uses. As far as the comedy is concerned, the address terms are especially efficient regarding the progress of the plot since they give the speaker a way to influence the addressee's actions. They indicate the rank of the different characters on a vertical scale and help to understand how the speaker views himself and the addressee (titles are especially efficient in this regard). A well chosen vocative may help to redress a FTA or reinforce a FFA. It also has a phatic function and contributes to maintaining contact between speaker and addressee. By naming the addressee, the speaker acknowledges and welcomes him. Let us add that, as the language register of the Latin comedy is close to conversational language, characters use generally one-word addresses, such as names or the second person pronoun $t u$. The tragic characters, on the other hand, have to keep with a mythical story whose outcome is ineluctable and well known to the audience. They usually choose long periphrases to summon the addressees, thus recalling their family history and their ancestors. They tend to use very formal address terms.

\section{AUTEUR}

\section{SOPHIE ROESCH}

Université François Rabelais (Tours) 